\title{
A CLASS OF PROJECTIVE PLANES OF CUBIC ORDER
}

\author{
PETER LORIMER
}

A class of projective planes is constructed which, in the finite case, have orders $q^{3}$ where $q$ is a power of a prime not equal to 2 or 3 . They are Veblen-Wedderburn planes but do not fit into any of the known systems of such planes.

Throughout the paper $F$ will be a field with characteristic not equal to 2 or $3, x^{3}-\alpha x-\beta$ will be a cubic irreducible over $F$, and $V$ will be the three dimensional vector space over $F$ consisting of triples of the form $(a, b, c), a, b, c \in F$.

Multiplication on the right in $V$ will be defined by means of the matrices of the form

$$
A_{a b c}=\left(\begin{array}{ccc}
a & b & c \\
\alpha b-\frac{1}{2} \beta c & a & -2 b \\
-\frac{1}{2} \beta b-\alpha^{2} c & -2 \beta c & a+2 \alpha c
\end{array}\right)
$$

and we will denote by $M$ the set of such matrices in which $a, b, c$ are not all zero.

Proposition 1. If $A_{a b c} \in M$ then $\operatorname{det}\left(A_{a b c}\right) \neq 0$.

Proof. The polynomial $x^{3}-\alpha x-\beta$ is irreducible over $F$. If $\lambda$ is a zero of this polynomial then, since $x^{3}-\alpha x-\beta$ is irreducible, $1, \lambda$ and $\lambda^{2}$ form a basis of the field $F(\lambda)$. Multiplication in $F(\lambda)$ is defined by the equation

$$
\begin{aligned}
(x+y \lambda & \left.+z \lambda^{2}\right)\left(a+b \lambda+c \lambda^{2}\right)=(x a+y \beta c+z \beta b) \\
& +(x b+y(a+\alpha c)+z(\alpha b+\beta c)) \lambda+(x c+y b+z(a+\alpha c)) \lambda^{2}
\end{aligned}
$$

for all $a, b, c, x, y, z$ in $F$. This equation is obtained by expanding the left-hand side and putting $\lambda^{3}=\alpha \lambda+\beta$.

Now multiplication on the right is a linear transformation and from the above equation the corresponding matrix is

$$
B_{a b c}=\left(\begin{array}{ccc}
a & b & c \\
\beta c & a+\alpha c & b \\
\beta b & \alpha b+\beta c & a+\alpha c
\end{array}\right) .
$$

A check now shows that $\operatorname{det}\left(A_{a b c}\right)=\operatorname{det}\left(B_{a b c}\right)$.

Received by the editors February 29, 1968. 
But multiplication on the right in the field $F(\lambda)$ is nonsingular so that $\operatorname{det}\left(B_{a b c}\right) \neq 0$.

Hence $\operatorname{det}\left(A_{a b c}\right) \neq 0$.

The next two propositions are verified by direct calculation.

Proposition 2. If $a, b, c, x, y, z \in F$ then $(a, b, c) A_{x y z}=(x, y, z) A_{a b c}$.

Proposition 3. If $a, b, c, x, y, z \in F$ then $A_{a b c}+A_{x y z}=A_{p q r}$ where $p=a+x, q=b+y, r=c+z$.

Proposition 4. If $u, v, w, x, y, z \in F, x, y, z$ not all zero, then there is $a$ unique $(a, b, c) \in V$ with the property $(x, y, z) A_{a b c}=(u, v, w)$.

Proof. By Proposition 1, $A_{x y z}$ is nonsingular so that there exists $(a, b, c) \in V$ with the property $(a, b, c) A_{x y z}=(u, v, w)$. Hence, by Proposition 2, $(x, y, z) A_{a b c}=(u, v, w)$.

If also $(x, y, z) A_{p q r}=(u, v, w)$ then $(x, y, z)\left(A_{p q r}-A_{a b c}\right)=0$, i.e. $(x, y, z) A_{d e f}=0$ where $d=p-a, e=q-b, f=r-c$.

Hence $A_{d e f}$ is singular which contradicts Proposition 1 unless $d=e$ $=f=0$.

This proves Proposition 4.

Theorem 1. Let $V$ be as above. Define right multiplication in $V$ by the equation

$$
(x, y, z)(a, b, c)=(x, y, z) A_{a b c} .
$$

Then $V$ is a Veblen-Wedderburn system, i.e.

(1) $V$ with addition is an abelian group.

(2) The nonzero elements of $V$ form a loop under multiplication.

(3) $(u+v) w=u w+v w$ for every $u, v$, w in $V$.

(4) If $u, v \in V, u \neq v$ then the equation $x u=x v+w$ has a unique solution in $V$.

See $[1$, p. 362].

Proof. (1) holds because $V$ is a vector space.

(2) We must show first that any two of the triples in the equation

$$
(x, y, z)(a, b, c)=(u, v, w)
$$

uniquely determines the other, under the condition that none of these triples is the zero vector.

Multiplication is well defined so that the first two determine the third uniquely.

If the first and third are given then the existence and uniqueness of the second is given by Proposition 4.

The remaining part follows from Propositions 3 and 4. 
Finally $1=(1,0,0)$ is an identity for $V$ since $v 1=1 v=v$ for every $v \in V$.

(3) follows because each $A_{a b c}$ is a linear transformation.

Before we prove (4) we notice that Proposition 2 implies that $u v=v u$ for every $u, v \in V$.

(4) Suppose $u, v \in V, u \neq v$.

Since $u-v \neq 0$, it follows from Proposition 4 that there is a unique $x=(a, b, c) \in V$ with the property $(u-v) A_{a b c}=w$, i.e. $x u=x v+w$.

If $y u=y v+w$ then $(u-v) y=w$ so that by the uniqueness of $x$, it follows that $y=x$.

This proves Theorem 1.

We now mention two other properties of the Veblen-Wedderburn system defined in Theorem 1 .

Theorem 2. Let $V$ be as in Theorem 1 and suppose $u, v, w \in V$. Then (1) $u v=v u$; (2) $u(v+w)=u v+u w$.

Proof. (1) follows directly from Proposition 2 and (2) follows from (1) and law (3) of Theorem 1.

We will prove now that $V$ is a proper Veblen-Wedderburn system, i.e., it is not a ternary ring for a Desarguesian plane. If it were, the multiplicative loop of $V$ would be a group and hence it is sufficient to prove

Proposition 5. $A_{010}^{-1} \notin M$.

Proof.

$$
A_{010}=\left(\begin{array}{ccc}
0 & 1 & 0 \\
\alpha & 0 & -2 \\
-\frac{1}{2} \beta & 0 & 0
\end{array}\right)
$$

so that

$$
\beta A_{010}^{-1}=\left(\begin{array}{ccc}
0 & 0 & -2 \\
\beta & 0 & 0 \\
0 & -\frac{1}{2} \beta & -\alpha
\end{array}\right) .
$$

It can easily be seen that $A_{010}^{-1} \notin M$.

It also follows from this proposition that $V$ is not an alternative division ring.

\section{REFERENCE}

1. Marshall Hall, Jr., The theory of groups, Macmillan, New York, 1961.

University of AUCKLaNd, New Zealand 